\title{
Gases de efecto invernadero generados por la extracción de oro. El ejemplo de la Mina Apumayo, Perú
}

\author{
Isabel Mercedes Solórzano Ortiz y Santiago Gualberto Valverde Espinoza \\ Universidad Nacional de Ingeniería Lima- Perú. \\ isolorzano.ortiz@gmail.com; svalverde@uni.edu.pe
}

\begin{abstract}
RESUMEN
En el Perú, a la fecha, no se han establecido metodologías que evalúen los efectos de las actividades productivas en el cambio climático, probablemente debido a que se no cuenta con investigación que demuestre estos efectos en el ámbito nacional. La minería es la que mayores avances en regulación ambiental ha tenido en los últimos años, ésta no cuenta con investigaciones enfocadas al cambio climático en casos específicos en el Perú. Sin embargo, existen herramientas de cálculo como la huella de carbono, que mide la cantidad total de gases de efecto invernadero (GEI) generados por una actividad y nos da un alcance de los impactos en el cambio climático de esta actividad o actividades. En ese sentido, el objetivo del estudio fue determinar la huella de carbono generada por las actividades de explotación de una mina de oro en el Perú, y así dar un alcance de los impactos de esta actividad en el cambio climático, tomando como base la explotación de un yacimiento aurífero conformado por rocas volcánicas y depósitos cuaternarios. Como resultado se obtuvo que, debido al significativo volumen de petróleo utilizado durante el transporte de mineral y desmonte mediante camiones volquete, la explotación de un yacimiento de oro en el Perú contribuye considerablemente con GEI y por ende al cambio climático. Las emisiones totales de GEI o huella de carbono por las actividades de producción de oro en la mina Apumayo en el año 2017 fueron de 24977.1 tCO 2 eq.
\end{abstract}

Palabras clave: depósitos cuaternarios, huella de carbono, minería, metalurgia.

\section{Greenhouse gases generated by gold mining. The example of the Apumayo Mine, Peru}

\section{ABSTRACT}

In Peru, there are no established methodologies to assess the effects of economic activities on climate change, probably because there is no research to demonstrate these effects at the national level. Even mining being an important economic activity in Peru and the one that has made the greatest progress in environmental regulation in recent years, it does not have research focused on climate change in specific cases. However, there are calculation tools, such as the carbon footprint, that measures the total amount of greenhouse gases (GHG) generated by an activity and gives us a scope of the climate change impacts of this activity or activities. In that sense, the objective of this study was to determine the carbon footprint generated by the exploitation activities of a gold mine in Peru, and thus give a first scope of the impacts on climate change, based on the exploitation of a goldfield formed by volcanic rocks and quaternary deposits. As a result, it was obtained that, due to the significant volume of petrol used in the transportation of ore and waste rock with heavy tippers, the exploitation activities of a gold mine in Peru contributes significantly to GHG and therefore to climate change. Total GHG emissions of carbon footprint from gold production activities at the Apumayo mine in 2017 were 24977.1 tCO2eq.

Keywords: carbon footprint, mining, metallurgy. 


\section{Introducción}

EI Perú es un país de bajas emisiones de GEI, con un porcentaje de participación mundial de solo 0.3 $\%$ sobre el total de emisiones. No obstante, presenta hasta siete de las nueve características reconocidas por la Convención Marco de las Naciones Unidas sobre el Cambio Climático (CMNUCC) para calificar como un país "particularmente vulnerable", debido a su alto grado de vulnerabilidad ante amenazas de origen hidro-meteorológico, como sequías y lluvias excepcionales, inundaciones, heladas, entre otros (MINAM, 2016).

A la fecha, en el Perú se cuentan con los Inventarios Nacionales de GEI los cuales han permitido identificar los sectores económicos con las mayores emisiones generadas y promover esfuerzos nacionales de gestión de emisiones (MINAM, 2018). Sin embargo, no se ha realizado una contabilización detallada de todas las emisiones de GEI de las actividades directas o indirectas que involucran a una organización o la producción de un bien o servicio, conocida como "huella de carbono".

Algunas empresas mineras han publicado de manera voluntaria (mediante sus reportes de sostenibilidad), las emisiones de GEI producidas de manera directa e indirecta por sus actividades (Minera Yanacocha S.R.L., Compañía Minera Antamina S.A. y Gold Fields La Cima S.A.), en términos de toneladas de dióxido de carbono equivalente. Sin embargo, estos reportes no muestran el detalle de la estimación de las emisiones, por lo que no permite la interpretación de los datos y tampoco permite realizar propuestas de mejora de la gestión ambiental minera.

Por ello la presente investigación, basada en la tesis para obtener el grado de maestro de Solórzano (2019), constituye el primer análisis de la "huella de carbono" de una unidad minera aurífera en el Perú, el cual bien puede ser usado como referente para próximas investigaciones y ampliar la información relacionada a este tema. Además, es importante mencionar que la tesis también presenta propuestas para la reducción o neutralización de las emisiones calculadas, con la aplicación de medidas de eficiencia energética y mejores prácticas ambientales.

\section{Objetivos}

1. Calcular la huella de carbono de la mina Apumayo, resultado de las emisiones de GEI generadas por todas las actividades (directas e indirectas) y áreas de soporte involucradas en la producción de las barras de oro.

2. Identificar las actividades con potencialidad de aplicación de mejoras para la reducción de la huella de carbono en la mina Apumayo.

\section{Zona de estudio}

El cálculo de la huella de carbono consideró todas las actividades ejecutadas en el año 2017, en la mina Apumayo, desde el minado hasta la obtención de las barras de oro.

\section{Localización}

Geopolíticamente, la mina Apumayo se ubica al sur de Perú, en el departamento de Ayacucho, distritos de Sancos y Chaviña; y, geológicamente se ubica sobre una secuencia de rocas del grupo barroso constituido por rocas volcánica de naturaleza lávica; y del grupo Tacaza formado por una secuencia estratigráfica de unidades sedimentarias, intercalaciones volcánico-sedimentarias, rocas volcánicas y depósitos cuaternarios recientes.

\section{Proceso Productivo}

La mina Apumayo realiza la explotación mediante el método de extracción a tajo abierto con voladura controlada de tres yacimientos (Apumayo, Huamanloma y Ayahuanca) con contenido de oro con reservas probadas 10.1 millones de toneladas de mineral. Seguidamente realiza el tratamiento del mineral extraído mediante el proceso metalúrgico de lixiviación en pilas con solución cianurada, para luego procesar la solución rica en la Planta Merrill Crowe hasta la obtención de las barras de oro.

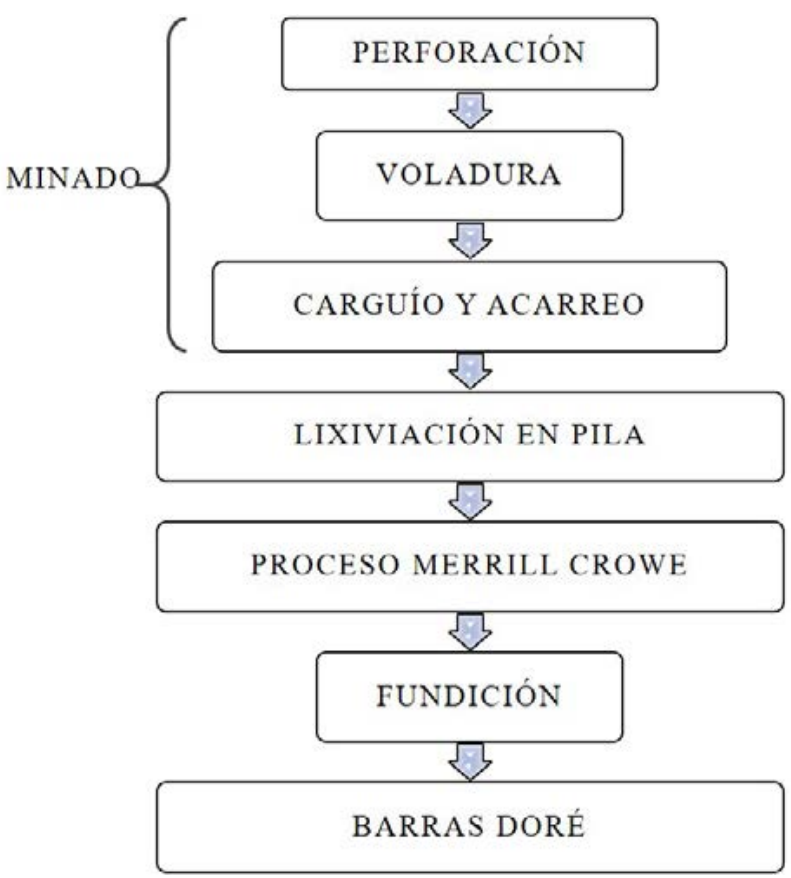

Figura 1. Etapas del Proceso de Producción de Oro en la mina Apumayo. (Mina Apumayo, 2018).

Figure 1. Gold Production Process at the Apumayo mine. (Mina Apumayo, 2018). 


\section{Minado.}

Consiste en la extracción del material que contiene oro en el yacimiento realizado por el método aTajo Abierto a un ritmo de 15000TM/día. Se realiza en dos fases:

- Perforación: Se perfora el terreno para colocar los explosivos y fragmentar el suelo para el carguío.

- Voladura: Los agujeros son Ilenados con material explosivo, que al detonar fragmentan la roca y remueven subterráneamente el material exponiéndolo a la superficie.

\section{Carguío y acarreo.}

Mediante camiones de 20 toneladas de capacidad, se transporta el mineral extraído del tajo a la pila de lixiviación (Pad) de un área aproximada de 47 hectáreas, acondicionada previamente mediante técnicas estrictas de impermeabilización. Asimismo, el material con baja ley que no resulta rentable de tratamiento se transporta como residuo mineral hacia el depósito de desmonte.

\section{Lixiviación en pila(Pad).}

El mineral descargado en la pila de lixiviación es lavado con solución cianurada para recuperar el oro con un flujo de riego de $600 \mathrm{~m}^{3} / \mathrm{h}$. La solución rica (cargada con oro) es llevada hacia la Poza de Solución Rica (PLS) para después de su tratamiento en la Planta Merrill Crowe, como solución pobre (sin oro), permanecer en la Poza de Solución Intermedia (ILS) y ser rebombeada a la pila de lixiviación (circuito cerrado).

\section{Proceso Merrill Crowe.}

Proceso al que es sometida la solución rica en oro. Primero es clarificada para reducir los sólidos en suspensión; luego se elimina el oxígeno para finalmente añadir polvo de zinc para precipitar el oro y pasar al

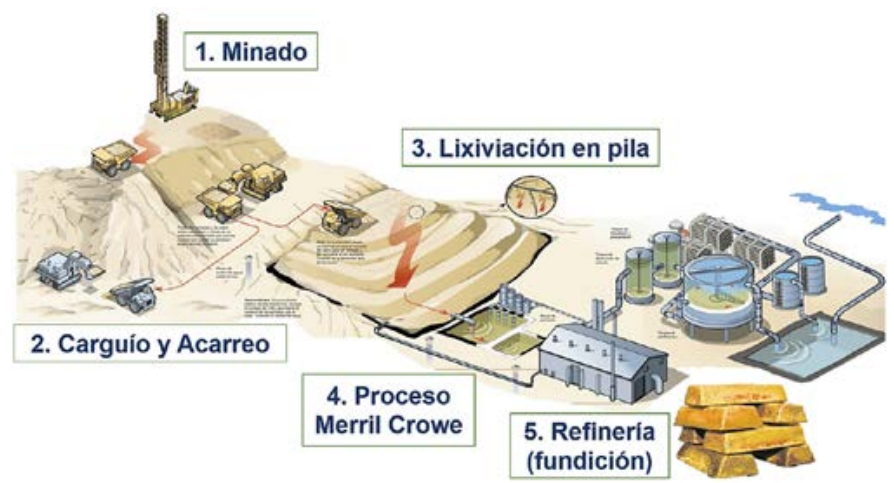

Figura 2. Esquema de la Producción de Oro en la mina Apumayo (Mina Apumayo, 2018).

Figure 2. Gold Production Scheme at the Apumayo mine (Mina Apumayo, 2018). proceso de refinería.

\section{Fundición.}

El precipitado de oro obtenido en el proceso Merrill Crowe es sometido a operaciones de secado en hornos de retortas a $650^{\circ} \mathrm{C}$. Finalmente, el producto obtenido pasa por un proceso de fundición para obtener las barras doré como producto final con una producción de 5000 onzas/mes.

\section{Limitaciones}

Dado que no existen factores de emisión para todos los materiales e insumos utilizados en el proceso productivo de la mina Apumayo, algunos valores de generación gases de efecto invernadero no pudieron ser contabilizados. Sin embargo, se considera la hipótesis de que estos valores serían despreciables comparado con la cantidad de $\mathrm{tCO}_{2}$ eq contabilizados en toda la mina Apumayo.

\section{Metodología}

La Huella de Carbono describe la cantidad de emisiones de GEl causados directa o indirectamente por una organización, producto o evento, a lo largo de su ciclo de vida. (COP20, 2015)

Se determina mediante un inventario de emisiones de GEI, medidas en toneladas de dióxido de carbono equivalente $\left(\mathrm{tCO}_{2}\right.$ eq) dado que el dióxido de carbono es el gas de efecto invernadero más significativo (Atlantic Consulting, 2009).

Para el cálculo de la Huella de Carbono de la mina Apumayo se utilizó la metodología GHG Protocol Alcance 3 , la cual incluye las emisiones directas, emisiones indirectas y otras emisiones indirectas de las actividades de la organización. Esta metodología es de fácil aplicación y tiene un amplio uso a nivel internacional, contando además con el respaldo de la USAID y de la USEPA.

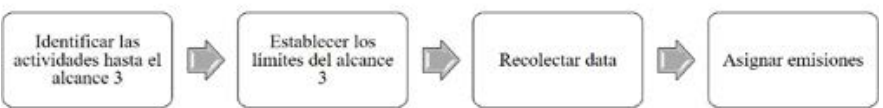

Figura 3. Pasos de la metodología GHG Protocol utilizados para el cálculo de huella de carbono de la mina Apumayo adaptado de (GHG Protocol, 2011, pág. 19).

Figure 3. Steps of GHG Protocol methodology used to calculate the carbon footprint of Apumayo mine, adapted from (GHG Protocol, 2011, pág. 19).

Paso 1: Para realizar la "identificación de las actividades hasta el alcance 3", se realizó un listado de todas las actividades desarrolladas dentro de cada 
proceso y área de la mina Apumayo y se las clasificó por su alcance:

\begin{tabular}{|c|c|c|}
\hline Proceso & Área asociada & Alcance \\
\hline Planeamiento & Geología & Alcances 1 y 3 \\
\hline Minado & Operaciones Mina & Alcances 1 y 3 \\
\hline Carguío y acarreo & Operaciones Mina & Alcances 1 y 3 \\
\hline Lixiviación en pila & Planta-Pad de lixiviación & Alcances 1,2 y 3 \\
\hline Proceso Merrill Crowe & Planta-Planta Merrill Crowe & Alcances 1,2 y 3 \\
\hline Fundición & Planta-Fundición & Alcances 2 y 3 \\
\hline \multirow{11}{*}{ Soporte } & Administración & Alcances 1,2 y 3 \\
\hline & Almacén & Alcances 1,2 y 3 \\
\hline & Seguridad de la propiedad & Alcance 3 \\
\hline & Laboratorio químico & Alcances 1,2 y 3 \\
\hline & Casa de fuerza & Alcance 1 \\
\hline & Departamento eléctrico & Alcance 1 \\
\hline & Taller mantenimiento mecánico & Alcances 1,2 y 3 \\
\hline & Centro médico & Alcances 1,2 y 3 \\
\hline & Ingeniería & Alcances 1 y 3 \\
\hline & Medio Ambiente & Alcances 1,2 y 3 \\
\hline & Comedor & Alcances 1,2 y 3 \\
\hline
\end{tabular}

Tabla 1. Alcances según la metodología GHG Protocol por proceso y área asociada de la mina Apumayo (Solórzano, 2019).

Table 1. Scopes according to the GHG Protocol methodology by process and department of Apumayo mine (Solórzano, 2019).

Paso 2: Se estableció el alcance de las actividades de todas las áreas involucradas en la operación de la mina Apumayo, siguiendo los lineamientos del GHG Protocol.

- Alcance 1: Emisiones provenientes de la combustión de combustibles fósiles como petróleo diésel en equipos motorizados y Gas Licuado de Petróleo (GLP) en las cocinas del comedor.

- Alcance 2: Emisiones por el uso de energía eléctrica adquirida del Sistema Eléctrico Interconectado Nacional (SEIN) en los equipos de procesamiento (lixiviación, proceso Merrill Crowe y fundición), así como en instalaciones auxiliares (oficinas, campamento, laboratorios, talleres, etc.).

- Alcance 3: Emisiones por el transporte y consumo de insumos y materiales y por la generación de residuos sólidos y su transporte al lugar de disposición final.

Paso 3: De acuerdo a los alcances definidos para cada uno de los procesos y áreas involucradas se recolectó la data con el apoyo de personal clave de la mina Apumayo.

Paso 4: Finalmente la asignación (cuantificación) de emisiones para cada tipo de alcance se realizó utilizando las siguientes fórmulas:

\section{Cuantificación de las emisiones de GEI del alcance 1}

Esta contabilidad sólo incluye las emisiones por consumo de combustibles fósiles en las diferentes áreas:

Ecuación 1. Cálculo de emisiones de GEI por combustión de Diésel B5 y GLP

Emisiones ${ }_{1}=\mathrm{CC} \times \mathrm{PCN} / 10^{6} \times\left(\mathrm{FE}_{\mathrm{CO} 2}+\mathrm{FE}_{\mathrm{CH} 4} \times \mathrm{PCG}_{\mathrm{CH} 4}+\mathrm{FE}_{\mathrm{N} 2 \mathrm{O}} \times \mathrm{P}_{\mathrm{N} 2 \mathrm{O}}\right)$

Dónde:

- Emisiones: Emisiones generadas de GEI por combustión (Diésel B5 y GLP), en $\mathrm{tCO}_{2}$ eq

- CC: Cantidad de combustible consumido, en kg

- PCN: Poder Calórico Neto del Combustible utilizado, en $\mathrm{KJ} / \mathrm{kg}$

- $\mathrm{FE}_{\mathrm{CO} 2} \mathrm{FE}_{\mathrm{CH} 4} \mathrm{FE}_{\mathrm{N2} 2 \mathrm{O}}$ : Factores de Emisión de $\mathrm{CO}_{2^{\prime}}$ $\mathrm{CH}_{4}, \mathrm{~N}_{2} \mathrm{O}$ del Combustible utilizado, en: $\mathrm{kgCO}_{2} /$ GJ, $\mathrm{kgCH}_{4} / \mathrm{GJ}$ y kgN2O/GJ, respectivamente.

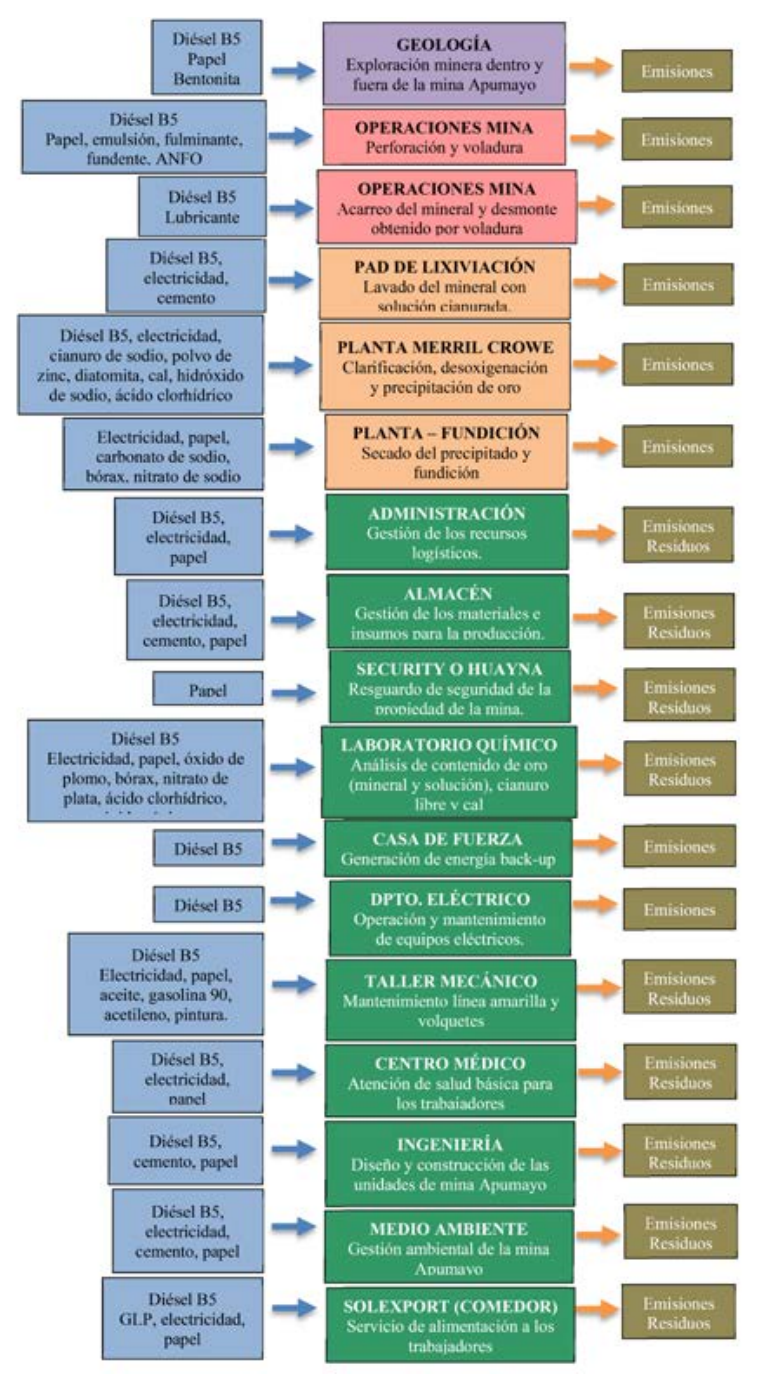

Figura 4. Ingresos y salidas en mina Apumayo (Solórzano, 2019). Figure 4. Inputs and outputs diagram of Apumayo mine (Solórzano, 2019). 
- $\quad \mathrm{PCG}_{\mathrm{CH}_{45}} \mathrm{PCG}_{\mathrm{N}_{2} \mathrm{O}}$ : Potencial de Calentamiento Global del $\mathrm{CH}_{4}$ y N $\mathrm{N}_{2} \mathrm{O}$ (21 y 310 respectivamente).

\begin{tabular}{|c|c|c|c|c|c|}
\hline $\begin{array}{c}\text { Tipo de } \\
\text { Combustible }\end{array}$ & $\begin{array}{c}\text { Poder } \\
\text { Calorifico } \\
\text { Neto }(\mathrm{KJ} / \mathrm{kg})\end{array}$ & $\begin{array}{c}\text { Densidad } \\
(\mathrm{kg} / \mathrm{L})\end{array}$ & $\begin{array}{c}\text { Factor de } \\
\text { emisión de } \\
\mathrm{CO}_{2} \\
(\mathrm{~kg} \\
\left.\mathrm{CO}_{2} / \mathrm{GJ}\right)\end{array}$ & $\begin{array}{c}\text { Factor de } \\
\text { emisión de } \\
\mathrm{CH}_{4} \\
(\mathrm{~kg} \\
\left.\mathrm{CH}_{4} / \mathrm{GJ}\right)\end{array}$ & $\begin{array}{c}\text { Factor de } \\
\text { emisión de } \\
\mathrm{N}_{2} \mathrm{O} \\
(\mathrm{kg} \\
\left.\mathrm{N}_{2} \mathrm{O} / \mathrm{GJ}\right)\end{array}$ \\
\hline Diésel B5 & 45500 & 0.87 & 74.1 & 0.004 & 0.004 \\
\hline GLP & 47300 & 0.542 & 63.1 & 0.062 & 0.0002 \\
\hline
\end{tabular}

Tabla 2. Valores de poder calorífico, densidad y factores de emisión de los combustibles REPSOL (2009), REPSOL (2011) e IPCC (1996). Table 2. Values of calorific value, density and fuel emission factors REPSOL (2009), REPSOL (2011) e IPCC (1996).

\section{Cuantificación de las emisiones de GEI del alcance 2}

Esta contabilidad sólo incluye las emisiones por consumo de energía eléctrica proveniente del Sistema Eléctrico Interconectado Nacional (SEIN).

Ecuación 2. Cálculo de emisiones de GEl por consumo de energía eléctrica

Emisiones $_{2}=$ Consumo de Energía $(\mathrm{MWh}) \times 0.547$ $\mathrm{tCO}_{2} / \mathrm{MWh}$

Donde:

- Emisiones: Emisiones generadas de GEI por consumo de energía, en $\mathrm{tCO}_{2}$ eq.

- Consumo de Energía: consumo de energía total reportado en la Unidad Minera, en MWh.

- $0.547 \mathrm{tCO}_{2} / \mathrm{MWh}$ : Factor de emisión de $\mathrm{CO}_{2}$ del SEIN para el año 2007 (FONAM citado por Ponce \& Rodríguez, 2016)

\section{Cuantificación de las emisiones de GEI del alcance 3}

Para el cálculo de las emisiones de GEI del alcance 3 en la mina Apumayo se consideraron tres aspectos:

- Consumo de insumos

- Generación de residuos sólidos

- Transporte de materiales e insumos y residuos sólidos

Ecuación 3. Cálculo de emisiones de GEI por consumo de insumos.

Emisiones $_{3,1}=\mathrm{CC}_{\mathrm{i}} \times \mathrm{FE}_{\mathrm{i}}$

Donde:

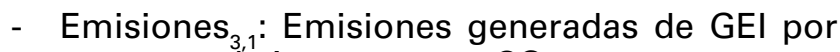
consumo de insumos, en $\mathrm{tCO}_{2}$ eq

- CC: Cantidad de insumo consumido, en kg o litros según corresponda.

- $\mathrm{FE}_{\mathrm{i}}$ : Factores de emisión de insumos (verTabla 3).

\begin{tabular}{|c|c|}
\hline Insumo & Factor de Emisión \\
\hline $\mathrm{Cal}^{1}$ & $1.22 \mathrm{kgCO}_{2} \mathrm{eq} / \mathrm{kg} \mathrm{de} \mathrm{Cal}$ \\
\hline Hidróxido de Sodio ${ }^{1}$ & $1.42 \mathrm{kgCO}_{2} \mathrm{eq} / \mathrm{kg} \mathrm{de} \mathrm{NaOH}$ \\
\hline Carbonato de Sodio $\left(\mathrm{Na}_{2} \mathrm{CO}_{3}\right)^{1}$ & $2.03 \mathrm{kgCO}_{2} \mathrm{eq} / \mathrm{kg} \mathrm{de} \mathrm{Na}_{2} \mathrm{CO}_{3}$ \\
\hline Cemento $^{1}$ & $0.10 \mathrm{kgCO}_{2} \mathrm{eq} / \mathrm{kg}$ de concreto \\
\hline $\mathrm{ANFO}^{2}$ & $0.18 \mathrm{kgCO}_{2} \mathrm{eq} / \mathrm{kg}$ de ANFO \\
\hline Emulsión ${ }^{2}$ & $0.17 \mathrm{kgCO}_{2} \mathrm{eq} / \mathrm{kg}$ de emulsión \\
\hline Fulminante $y$ fundente ${ }^{3}$ & $\begin{array}{l}0.17 \mathrm{kgCO}_{2} \mathrm{eq} / \mathrm{kg} \text { de fulminante } \mathrm{o} \\
\text { fundente }\end{array}$ \\
\hline Lubricante $^{2}$ & $0.081 \mathrm{kgCO}_{2} \mathrm{eq} / \mathrm{kg}$ de lubricante \\
\hline Aceite $^{2}$ & $0.08148 \mathrm{kgCO}_{2} \mathrm{eq} / \mathrm{L}$ de aceite \\
\hline Gasolina $90^{2}$ & $0.18126 \mathrm{kgCO}_{2} \mathrm{eq} / \mathrm{L}$ de gasolina \\
\hline Papel' $^{1}$ & 0.00184 ton $\mathrm{CO}_{2} \mathrm{eq} / \mathrm{kg}$ de papel \\
\hline
\end{tabular}

Tabla 3. Factores de emisión de insumos 'Sotware GaBi (Bustos Donoso, 2011), ${ }^{2}$ NGA Factors En Tabla 4 (Explosive use) (NGA, 2008) $\mathrm{y}^{3}$ (Gold Fields, 2010).

Table 3. Supplies emission factors 'Sotware GaBi (Bustos Donoso, 2011), ${ }^{2}$ NGA Factors. In Table 4 (Explosive use) (NGA, 2008) y ${ }^{3}$ (Gold Fields, 2010).

Ecuación 4. Cálculo de emisiones de GEI por generación de residuos sólidos.

Emisiones $_{3,2}=\mathrm{CC}_{\mathrm{r}} \times \mathrm{FE}_{\mathrm{a}}$

Donde:

- Emisiones ${ }_{3,2}$ : Emisiones generadas de GEI por generación de residuos sólidos, en $\mathrm{tCO}_{2} \mathrm{eq}$

- CC: Cantidad de residuo sólido generado según tipo, en TN.

- $\mathrm{FE}_{\mathrm{a}}$ : Factores de Emisión por tipo de residuo sólido (verTabla 4).

\section{Tipo de residuo Factor de emisión}

$\left(\mathrm{tCO}_{2} \mathrm{eq} /\right.$ ton residuo)

\begin{tabular}{|c|c|}
\hline Papel y cartón & 2.5 \\
\hline Orgánicos & 0.9 \\
\hline Vidrios & 0 \\
\hline Metálicos & 0 \\
\hline Plásticos & 0 \\
\hline Peligrosos & 0.3 \\
\hline Generales * & 0.9 \\
\hline
\end{tabular}

Tabla 4. Factores de emisión por tipo de residuo sólido $(*)$ Mezclados es equivalencia con la nomenclatura extranjera. Fuente: (NGA, 2008).

Table 4. Emission factors by type of solid waste . 
Ecuación 5. Cálculo de consumo de combustible de vehículos de transporte

$$
\mathrm{CC}=\text { Distancia }_{\mathrm{i}} \times \text { Frecuencia }_{\mathrm{i}} \times \text { Consumo }
$$

Dónde:

- CC: Consumo de combustible total (gal/año)

- Distancia: tramo recorrido para el transporte del material, insumo o residuo i en $(\mathrm{km})$

- Frecuencia: Número de veces en las que se transporta el material, insumo o residuo i al año

- Consumo: Consumo promedio de combustible del vehículo (gal $/ \mathrm{km})$

\section{Resultados}

Las emisiones totales de GEI de la mina Apumayo en el año 2017 fueron de $24977.1 \mathrm{tCO}_{2}$ eq, siendo que $14869.2 \mathrm{tCO}_{2}$ eq $(60 \%)$ corresponden a emisiones directas de alcance 1 , un total de $8178.2 \mathrm{tCO}_{2}$ eq (33\%) corresponden a emisiones indirectas de alcance 2, y además $1929.7 \mathrm{tCO}_{2}$ eq (7\%) corresponden a emisiones indirectas de alcance 3 .

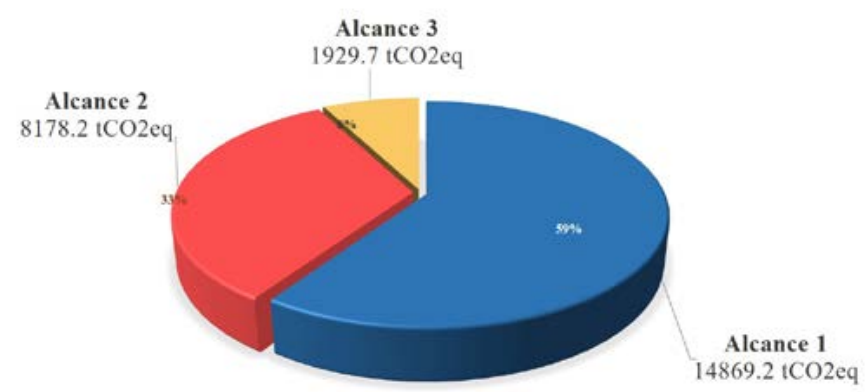

Figura 5. Emisiones según alcance de la mina Apumayo. (Solórzano, 2019)

Figure 5. Emissions by scope of Apumayo mine. (Solórzano, 2019).

En la Tabla 5. se presentan los consumos de la mina Apumayo para en todo el proceso del oro y el inventario de GEI por cada uno de ellos.

\section{Alcance 1: Emisiones directas de GEI.}

El mayor porcentaje de estas emisiones con aproximadamente $68 \%$ corresponden al proceso de carguío y acarreo por el consumo de diésel en camionetas, equipos pesados (volquetes, excavadoras, equipos auxiliares) y camiones cisterna. En segundo lugar, están las actividades de perforación y voladura con un $15.7 \%$ por el consumo de diésel en perforadoras, camión fábrica, camionetas, entre otros; y en tercer lugar se encuentra el área de soporte con un $13 \%$ por el consumo de diésel en camionetas y equipos auxiliares.

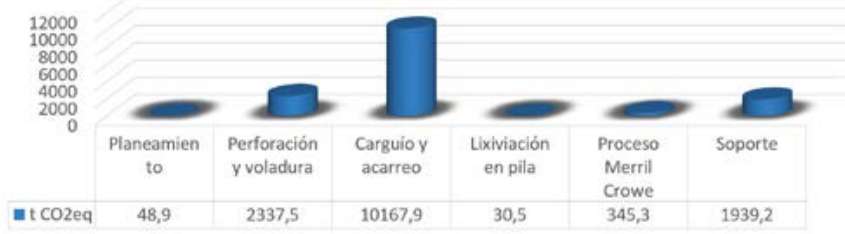

Figura 6. Emisiones de alcance 1 por proceso de la mina Apumayo (Solórzano, 2019).

Figure 6. Scope 1 emissions by process of Apumayo mine (Solórzano, 2019).

\section{Alcance 2: Emisiones indirectas de GEI.}

El mayor porcentaje de estas emisiones, aproximadamente $54 \%$, corresponden al proceso de lixiviación en pila, seguido por el proceso Merrill Crowe con un $37 \%$, en tercer lugar, el área de soporte con un $8 \%$, y por último el proceso de fundición con tan sólo el $1 \%$.

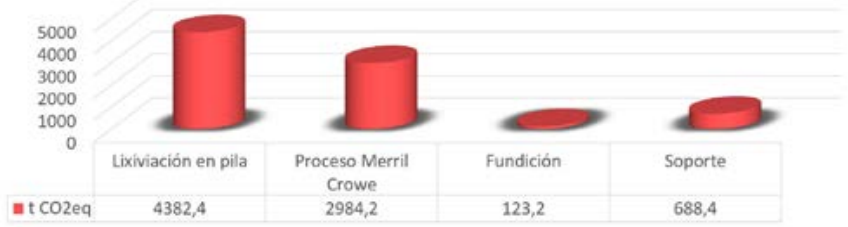

Figura 7. Emisiones de alcance 2 por proceso de la mina Apumayo. (Solórzano, 2019).

Figure 7. Scope 2 emissions by process in Apumayo mine. (Solórzano, 2019).

\section{Alcance 3: Otras emisiones indirectas.}

Las mayores emisiones corresponden al proceso de minado con aproximadamente $45 \%$ debido al consumo de insumos para las actividades de perforación y voladura, en segundo lugar, el área de soporte con un $32 \%$ debido al transporte de materiales, insumos y residuos sólidos, así como a la generación de residuos sólidos orgánicos en el comedor y por último, el proceso de Merrill Crowe con un 22\% debido al consumos de insumos para la clarificación, desoxigenación y precipitación del oro.

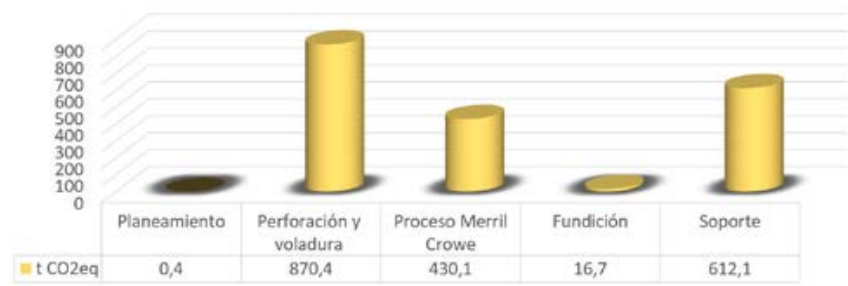

Figura 8. Emisiones de alcance 3 por proceso de la mina Apumayo (Solórzano, 2019).

Figure 8. Scope 3 emissions by process of Apumayo mine (Solórzano, 2019). 


\begin{tabular}{|c|c|c|c|c|c|c|c|c|c|c|c|}
\hline PROCESO & $\begin{array}{c}\text { ÁREA } \\
\text { ASOCIADA }\end{array}$ & $\begin{array}{l}\text { ACION DE CONSL } \\
\text { ACTIVIDADES } \\
\text { PRINCIPALES }\end{array}$ & $\begin{array}{l}\text { UMOS DE LA UNIDIDADES } \\
\text { SECUNDARIAS }\end{array}$ & $\begin{array}{l}\text { AD MINERA } \\
\text { ALCANCE }\end{array}$ & $\begin{array}{l}\text { APUMAYO PA } \\
\text { CONSUMO }\end{array}$ & $\begin{array}{c}\text { ARA CALC } \\
\text { UNIDAD } \\
1\end{array}$ & $\begin{array}{l}\text { CULO DE EMIS } \\
\text { CONSUMO }\end{array}$ & UNIDAD & $\begin{array}{l}\text { E GEI - ANO } 2 \\
\text { CONSUMO }\end{array}$ & $\begin{array}{l}\text { UNIDAD } \\
\text { FINAL }\end{array}$ & t co2eq \\
\hline \multirow{4}{*}{ Plancamicnto } & \multirow{4}{*}{ Geologia } & \multirow{4}{*}{\begin{tabular}{|} 
Exploración minera \\
dentro y fuera \\
(áreas de \\
ampliación) de la \\
Unidad Minera \\
Apurnayo
\end{tabular}} & $\begin{array}{l}\text { Combustible dićscl } \\
\text { on camionotas y } \\
\text { maquinaria } \\
\text { perforadora }\end{array}$ & Alcance 1 & $4,322.6$ & gal & $16,363.0$ & $L$ & $14,235.8$ & $\mathrm{~kg}$ & 48.9 \\
\hline & & & Papel & Alcance 3 & 128.6 & $\mathrm{~kg}$ & & & & & 0.2 \\
\hline & & & Bentonita & Alcance 3 & 4.500 .0 & $\mathrm{~kg}$ & & & & & N.D. \\
\hline & & & $\begin{array}{l}\text { Generación de } \\
\text { residuos de papel y } \\
\text { cartón }\end{array}$ & Alcance 3 & 73.6 & $\mathrm{~kg}$ & 0.1 & fon & & & 0.2 \\
\hline \multirow{6}{*}{ Minado } & \multirow{6}{*}{$\begin{array}{l}\text { Operaciones } \\
\text { Mina }\end{array}$} & \multirow{6}{*}{$\begin{array}{l}\text { Perforación y } \\
\text { voladura }\end{array}$} & $\begin{array}{l}\text { Combustible diésel } \\
\text { en camionetas y } \\
\text { equipos pesados de } \\
\text { perforación y } \\
\text { voladura. }\end{array}$ & Alcance 1 & $206,819.2$ & gal & $782,895.5$ & $L$ & $681,119.1$ & $\mathrm{~kg}$ & $2,337.5$ \\
\hline & & & Papel & Alcance 3 & 84.2 & $\mathrm{~kg}$ & & & & & 0.2 \\
\hline & & & Emulsión & Alcance 3 & $1,695,205.0$ & $\mathrm{~kg}$ & & & & & 288.2 \\
\hline & & & Fulminante & Alcance 3 & $3,685.5$ & $\mathrm{~kg}$ & & & & & 0.6 \\
\hline & & & \begin{tabular}{l|} 
Fundente \\
premezclado
\end{tabular} & Alcance 3 & $10,075.0$ & $\mathrm{~kg}$ & & & & & 1.7 \\
\hline & & & $\begin{array}{l}\text { Nitrato de amonio } \\
\text { (ANFO) }\end{array}$ & Alcance 3 & $3,220,745.0$ & $\mathrm{~kg}$ & & & & & 579.7 \\
\hline \multirow[t]{2}{*}{$\begin{array}{l}\text { Carguilo y } \\
\text { acarreo }\end{array}$} & \multirow[t]{2}{*}{$\begin{array}{l}\text { Operaciones } \\
\text { Mina }\end{array}$} & \multirow[t]{2}{*}{$\begin{array}{l}\text { Acarreo del minera } \\
\text { y desmonte } \\
\text { obtenido posterior } \\
\text { a la voladura }\end{array}$} & $\begin{array}{l}\text { Combustible diésel } \\
\text { en camionotas y } \\
\text { cquipos posados } \\
\text { (volquctos, } \\
\text { excavadoras, } \\
\text { equipos auxiliares) }\end{array}$ & Alcance 1 & $899,661.5$ & gal & $3,405,587.6$ & L & $2,962,861.2$ & $\mathrm{~kg}$ & $10,167.9$ \\
\hline & & & Iubricante & Alcance 3 & 5.0 & gal & 18.9 & L & & & 0.0 \\
\hline \multirow{3}{*}{$\begin{array}{l}\text { Lixiviación en } \\
\text { pila }\end{array}$} & \multirow{3}{*}{$\begin{array}{l}\text { Planta Pad do } \\
\text { lixiviación }\end{array}$} & \multirow{3}{*}{$\begin{array}{l}\text { Lavado del mineral } \\
\text { con solución } \\
\text { cianurada }\end{array}$} & $\begin{array}{l}\text { Combustible diésel } \\
\text { por camionetas y } \\
\text { cquipos posados }\end{array}$ & Alcance 1 & 2.701 .5 & gal & 10.226 .3 & $\mathrm{~L}$ & 8.896 .9 & $\mathrm{~kg}$ & 30.5 \\
\hline & & & Energía electrica & Alcance 2 & $8,011,787.6$ & kWh/año & $8,011.8$ & 8) MWhaño & & & $4,382.4$ \\
\hline & & & Cemento & Alcance 3 & 85.0 & $\mathrm{~kg}$ & & & & & 0.0 \\
\hline \multirow{8}{*}{$\begin{array}{c}\text { Proceso } \\
\text { Merril Crowe }\end{array}$} & \multirow{8}{*}{$\begin{array}{l}\text { Planta-Planta } \\
\text { Merril Crowe }\end{array}$} & \multirow{8}{*}{$\begin{array}{l}\text { Clarificación, } \\
\text { desoxigenación y } \\
\text { precipitación de } \\
\text { oro }\end{array}$} & $\begin{array}{l}\text { Combustible diésel } \\
\text { en camionetas y } \\
\text { equipos pesados }\end{array}$ & Alcance 1 & $30,552.9$ & gal & $115,655.3$ & $\mathrm{~L}$ & $100,620.1$ & $\mathrm{~kg}$ & 345.3 \\
\hline & & & Energla eléctrica & Alcance 2 & $5,455,558.7$ & kWh/año & $5,455.6$ & 6) MWh/año & & & $2,984.2$ \\
\hline & & & Cianuro de sodio & Alcance 3 & $340,500.0$ & $\mathrm{~kg}$ & & & & & N.D. \\
\hline & & & Polvo de zinc & Alcance 3 & $175,000.0$ & $\mathrm{~kg}$ & & & & & N.D. \\
\hline & & & Diatomila & Alcance 3 & $49,986.7$ & $\mathrm{~kg}$ & & & & & N.D. \\
\hline & & & Oxido de calcio (cal) & Alcance 3 & $300,000.0$ & $\mathrm{~kg}$ & & & & & 366.0 \\
\hline & & & Hidróxido de sodio & Alcancc 3 & 45.160 .0 & $\mathrm{~kg}$ & & & & & 64.1 \\
\hline & & & $\begin{array}{l}\text { Acido clorhídrico } \\
\text { industrial }\end{array}$ & Alcance 3 & $1,440.0$ & $\mathrm{~kg}$ & & & & & N.D. \\
\hline \multirow{5}{*}{ Fundición } & \multirow{5}{*}{$\begin{array}{l}\text { Planta- } \\
\text { Refinería }\end{array}$} & \multirow{5}{*}{$\begin{array}{l}\text { Secado del } \\
\text { precipitado y } \\
\text { fundición }\end{array}$} & Energía eléctrica & Alcance ? & $225,238.7$ & kWh/año & 225.2 & 2) MWh/año & & & 123.2 \\
\hline & & & Papel & Alcance 3 & 39.8 & $\begin{array}{ll}\mathrm{kg} \\
\end{array}$ & & & & & 0.1 \\
\hline & & & Carbonato de sodio & Alcance 3 & $8,175.0$ & $\mathrm{~kg}$ & & & & & 16.6 \\
\hline & & & Bórax & Alcance 3 & $17,410.6$ & $\mathrm{~kg}$ & & & & & N.D. \\
\hline & & & Nitrato de sodio & Alcance 3 & $2,363.0$ & $\mathrm{~kg}$ & & & & & N.D. \\
\hline & \multirow{8}{*}{ Administración } & \multirow{8}{*}{$\begin{array}{c}\text { Administración de } \\
\text { los recursos } \\
\text { logísticos }\end{array}$} & $\begin{array}{l}\text { Combustible dlésel } \\
\text { en vehiculos de } \\
\text { transporte de } \\
\text { empleados }\end{array}$ & Alcanco 1 & $33,836.6$ & gal & 128.085 .4 & $\mathrm{~L}$ & 111.434 .3 & $\mathrm{~kg}$ & 382.4 \\
\hline & & & \begin{tabular}{|l|} 
Energía eléctrica en \\
el camparnento y \\
oficinas \\
administrativas
\end{tabular} & Alcanco 2 & $543,435.1$ & $\mathrm{kWh} / \mathrm{año}$ & 543.4 & 4. MWh/año & & & 297.3 \\
\hline & & & \begin{tabular}{|l|} 
Cornbustible de \\
camiones cisterna \\
para el transporte de \\
agua de consumo \\
doméstico
\end{tabular} & Alcance 3 & 427.4 & gal & $1,617.9$ & L & $1,407.6$ & $\mathrm{~kg}$ & 4.8 \\
\hline & & & Papel & Alcance 3 & 851.4 & $\mathrm{~kg}$ & & & & & 1.6 \\
\hline & & & \begin{tabular}{|l|} 
Generación de \\
residuos de vidrio
\end{tabular} & Alcance 3 & 473.0 & $\mathrm{~kg}$ & & & & & 0.0 \\
\hline & & & $\begin{array}{l}\text { Generación de } \\
\text { residuos melálicos }\end{array}$ & Alcancc 3 & $30,407.0$ & $\mathrm{~kg}$ & & & & & 0.0 \\
\hline & & & $\begin{array}{l}\text { Generación de } \\
\text { residuos plásticos }\end{array}$ & Alcance 3 & $1,289.5$ & $\mathrm{~kg}$ & & & & & 0.0 \\
\hline & & & $\begin{array}{l}\text { Generación de } \\
\text { residuos de papel y } \\
\text { cartón }\end{array}$ & Alcance 3 & 472.5 & $\mathrm{~kg}$ & 0.5 & ton & & & 1.2 \\
\hline
\end{tabular}




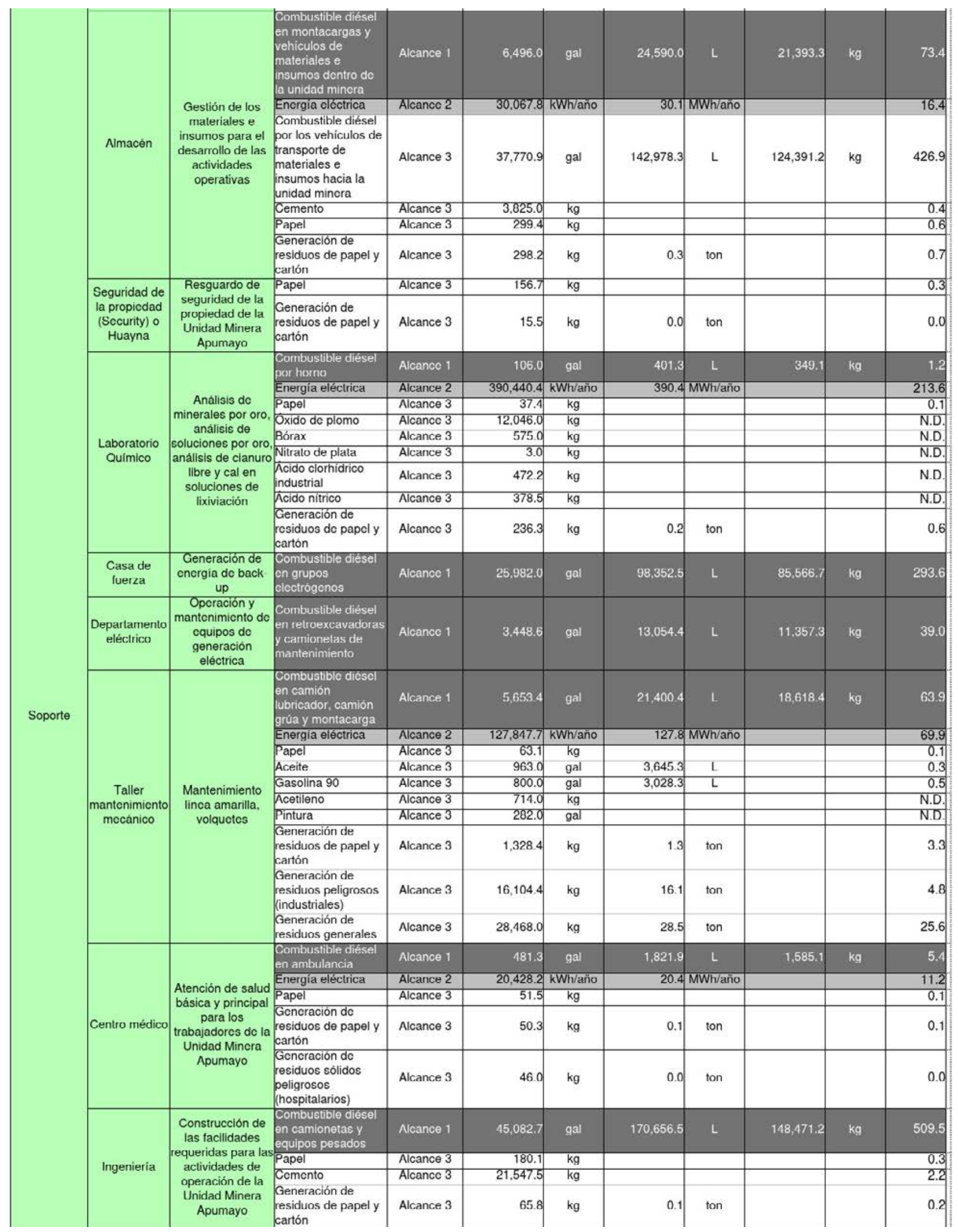


Solórzano Ortiz,T.M., 2021. Gases de efecto invernadero generados por la extracción de oro. El ejemplo de la Mina Apumayo, Perú, 132 (4): 583-5902

\begin{tabular}{|c|c|c|c|c|c|c|c|c|c|c|}
\hline \multirow{6}{*}{$\begin{array}{c}\text { Medio } \\
\text { Ambiente }\end{array}$} & \multirow{6}{*}{$\begin{array}{l}\text { Gestión ambiental } \\
\text { de la Unidad } \\
\text { Minera Apumayo }\end{array}$} & $\begin{array}{l}\text { Combustible en } \\
\text { equipo pesado } \\
\text { (retroexcavadora, } \\
\text { tractor, } \\
\text { motoniveladora. } \\
\text { entro otros) }\end{array}$ & Alcance 1 & $39,479.1$ & gal & $149,444.4$ & L & $130,016.7$ & $\mathrm{~kg}$ & 446.2 \\
\hline & & $\begin{array}{l}\text { Energia eléctrica por } \\
\text { Plantas de } \\
\text { Tratamionto do } \\
\text { Aguas Rosidualos }\end{array}$ & Alcance 2 & $49,595.4$ & $\mathrm{kWh} /$ año & 49.6 & MWh/año & & & 27.1 \\
\hline & & Cemento & Alcancc 3 & 170.0 & $\mathrm{~kg}$ & & & & & 0.0 \\
\hline & & Papel & Alcance 3 & 63.1 & $\mathrm{~kg}$ & & & & & 0.1 \\
\hline & & $\begin{array}{l}\text { Generación de } \\
\text { residuos de papel y } \\
\text { cartón }\end{array}$ & Alcance 3 & 58.1 & $\mathrm{~kg}$ & 0.1 & ton & & & 0.1 \\
\hline & & $\begin{array}{l}\text { Combustible en } \\
\text { transporte de } \\
\text { residuos hacia lugar } \\
\text { de disposición final }\end{array}$ & Alcance 3 & 966.4 & gal & $3,658.2$ & L & $3,182.7$ & $\mathrm{~kg}$ & 10.9 \\
\hline \multirow{7}{*}{$\begin{array}{l}\text { Solexport } \\
\text { (Comedor) }\end{array}$} & \multirow{7}{*}{$\begin{array}{l}\text { Provisión del } \\
\text { servicio de } \\
\text { alimentación a los } \\
\text { trabajadores }\end{array}$} & $\begin{array}{l}\text { Gas natural para } \\
\text { cocina }\end{array}$ & Alcance 1 & $18,702.0$ & gal & $70,794.7$ & $L$ & $38,370.7$ & kg & 117.0 \\
\hline & & $\begin{array}{l}\text { Combustible diésel } \\
\text { por horno de } \\
\text { panaderia }\end{array}$ & Alcance 1 & 661.0 & gal & 2.502 .2 & L & 2.176 .9 & $\mathrm{~kg}$ & 7.5 \\
\hline & & Energía eléctrica & Alcance 2 & $96,621.8$ & kWh/año & 96.6 & MWh/año & & & 52.9 \\
\hline & & $\begin{array}{l}\text { Combustible en } \\
\text { vehículos de } \\
\text { transporte de } \\
\text { insumos }\end{array}$ & Alcance 3 & $2,055.0$ & gal & $7,779.0$ & L & $6,767.7$ & $\mathrm{~kg}$ & 23.2 \\
\hline & & Papel & Alcance 3 & 39.8 & $\mathrm{~kg}$ & & & & & 0.1 \\
\hline & & $\begin{array}{l}\text { Generación de } \\
\text { residuos de papel y } \\
\text { cartón }\end{array}$ & Alcance 3 & $1,274.2$ & $\mathrm{~kg}$ & 1.3 & ton & & & 3.2 \\
\hline & & $\begin{array}{l}\text { Generación de } \\
\text { residuos sólidos } \\
\text { orgánicos }\end{array}$ & Alcance 3 & $110,583.0$ & $\mathrm{~kg}$ & 110.6 & ton & & & 99.5 \\
\hline
\end{tabular}

Tabla 5. Consumo por alcance - Inventario de GEI de la mina Apumayo.

Table 5. Consumption by scope - GHG inventory of the Apumayo mine.

\section{Discusión}

En la mina Apumayo, el proceso productivo que presentó mayor cantidad de emisiones fue el de carguío y acarreo, alcanzando las $10167.9 \mathrm{tCO}_{2}$ eq y $41 \%$ de las emisiones de GEl totales, por el consumo de gran cantidad de combustible para el funcionamiento de camionetas, equipos pesados (volquetes, excavadoras, equipos auxiliares) y camiones cisterna para el riego de vías.

Por otro lado, los procesos de lixiviación en pila y de Merrill Crowe también tienen un alto porcentaje de emisiones de GEI por su alta demanda eléctrica, representando en conjunto el $33 \%$ de las emisiones totales.

Por último, las actividades del área de soporte, representaron un $13 \%$ de las emisiones de GEI totales, debido al consumo de combustible para el transporte de personal, materiales e insumos; el consumo de energía eléctrica en el campamento, centro médico y otras áreas auxiliares, así como las emisiones por la generación de residuos sólidos y su transporte hacia el lugar de disposición final.

Debido a que, en el año 2017, las emisiones totales de GEI fueron de $24977.1 \mathrm{tCO}_{2}$ eq y la producción de oro 60185 onzas troy (1.88 toneladas), se tiene que la ratio de emisión de GEI de la mina Apumayo fue de $13150.6 \mathrm{tCO}_{2} \mathrm{eq} / \mathrm{t} \mathrm{Au}$ producido.

Las emisiones obtenidas en la mina Apumayo fue- ron comparados con los resultados presentados en los reportes de sostenibilidad de minas de oro similares como Yanacocha y Cerro Corona de Gold Fields.

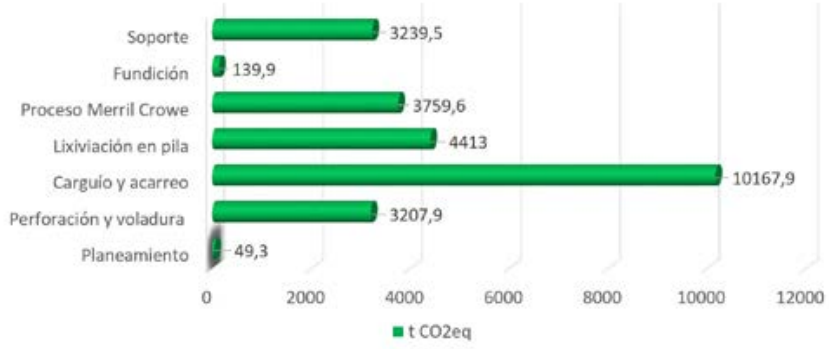

Figura 9. Emisiones totales por proceso de la mina Apumayo. (Solórzano, 2019).

Figure 9. Total emissions by process of Apumayo mine. (Solórzano, 2019).

\begin{tabular}{cccccc}
\hline Minera & $\begin{array}{c}\text { Año de } \\
\text { cálculo }\end{array}$ & $\begin{array}{c}\text { Producción } \\
\text { de } \mathrm{Au} \\
\text { (onzas/año) }\end{array}$ & $\begin{array}{c}\text { Producción } \\
\text { de } \mathrm{Au} \\
(\mathrm{t} / \mathrm{año})\end{array}$ & $\begin{array}{c}\text { Emisiones } \\
\text { totales de GEI } \\
\left(\mathrm{tCO}_{2} \mathrm{eq}\right)\end{array}$ & $\begin{array}{c}\text { Ratio de } \\
\text { emisión de GEI } \\
\left(\mathrm{tCO}_{2} \mathrm{eq} / \mathrm{t} \mathrm{Au}\right)\end{array}$ \\
\hline Yanacocha & 2013 & 1017000 & 31.63 & 583300.0 & 18441 \\
\hline $\begin{array}{c}\text { Cerro } \\
\text { Corona }\end{array}$ & 2016 & 150210 & 4.67 & 80577.0 & 17254 \\
\hline Apumayo & 2017 & 60185 & 1.87 & 24977.1 & 13357
\end{tabular}

Tabla 6. Ratio de emisión de GEI por unidad de producto para diferentes mineras de oro. Fuente: (Solórzano, 2019). Equivalencia: 1 tonelada $=32150.7$ onzas troy.

Table 6. GHG emission ratio per unit of product for different gold mines. 


\section{Conclusiones}

- Las emisiones totales de GEI o huella de carbono por las actividades de producción de oro en la mina Apumayo en el año 2017 fueron de $24977.1 \mathrm{tCO}_{2} \mathrm{eq}$.

- La ratio de emisión de GEI de la mina Apumayo en el año 2017 fue de 13357 tCO eq/t Au producido, por debajo de lo presentado en los reportes de sostenibilidad de Yanacocha (18441 $\left.\mathrm{tCO}_{2} \mathrm{eq} / \mathrm{t} \mathrm{Au}\right)$ y Cerro Corona de Gold Fields (17254 tCO 2 eq/t Au).

- El proceso de carguío y acarreo representó el $41 \%$ de las emisiones totales de GEl de la mina Apumayo, principalmente por el consumo de combustible en vehículos pesados como volquetes, tractores, cargadores frontales.

- Existen diferentes estrategias que pueden ser implementadas para reducir la huella de carbono en una mina de producción de oro. En principio se deben implementar indicadores de ecoeficiencia para lograr una gestión eficiente en la organización y de esta manera reducir actividades innecesarias que incrementen los GEI.

\section{Recomendaciones}

- Ampliar el análisis de la presente investigación estudio para un mayor periodo, por ejemplo, de los últimos 5 años $y$, de ser posible, para todo el ciclo de vida de la mina Apumayo, incluyendo también las etapas de construcción y cierre.

- Plantear estrategias para la reducción de los GEI en los procesos donde registraron los mayores valores. Las estrategias pueden considerar medidas de eficiencia en el uso de recursos, cambio de tecnología, optimización de los procesos, permuta del tipo combustible (por ejemplo, petróleo a gas natural), entre otros.

- Realizar mayores investigaciones en cuanto a Huella de Carbono relacionadas a proyectos de inversión en el Perú, con el fin de contar con una buena data de información que permita medir el desempeño de cada actividad productiva.

\section{Referencias}

Atlantic Consulting. 2009. Huella de Carbono del GLP en relación a otros Combustibles.

Bustos Donoso, J. F. 2011. Análisis de la Huella de Carbono en una Empresa Minera del Cobre en Chile. Santiago de Chile: Pontificia Universidad Católica de Chile.

COP20, L. 2015. LIMA COP20. Recuperado el 2018, de ¿Cómo se mide la Huella de Carbono?: http:// cop20.minam.gob.pe/22973/como-se-mide-la-hueIla-de-carbono/

FONAM, R. R. \& Rodríguez, D. A. 2016. Determinación de la Huella de Carbono del Country Club el Bosque - Sede Chosica". Trabajo deTitulación para Optar el Título de Ingeniero Forestal. Universidad Nacional Agraria la Molina. Lima.

GHG Protocol. 2011. Corporate Value Chain (Scope 3) Accounting and Reporting Standard. World Resources Institute and World Business Council for Sustainable Development.

Gold Fields. 2010. Carbon Footprint Report. Financial and Calendar year 2009. . Promethium Carbon (Pty) Ltd.

Mina Apumayo. 2018. Esquema de la Producción de Oro en la mina Apumayo, Perú.

MINAM. 2016. La Contribución Nacional del Perú iNDC: agenda para un desarrollo climáticamente responsable. Lima: Ministerio del Ambiente.

MINAM. 2018. INVENTARIO NACIONAL DE GASES DE EFECTO INVERNADERO. Obtenido de INFOCARBONO: http://infocarbono.minam.gob.pe/inventarios-nacionales-gei/intro/

NGA. 2008. National Greenhouse Accounts (NGA) Factors. Australia: Commonwealth of Australia

Solórzano, I. 2019. Estimación de la Huella de Carbono en la Unidad Minera Apumayo para el año 2017 y Propuestas para su Incorporación dentro del Estudio de Impacto Ambiental detallado. (Tesis de Maestría). Universidad Nacional de Ingeniería, Lima.

Recibido: julio 2020

Revisado: diciembre 2020

Aceptado: enero 2021

Publicado: diciembre 2021 